\title{
Nursing students' perception of simulation as a clinical teaching method in the Cape Town Metropole, South Africa
}

\author{
N Nel, BCur, MCur, PGND: Health Assessment, Treatment and Care, PGND: Management, PGND: Education; E L Stellenberg, DCur, RN, RM
}

Division of Nursing, Faculty of Medicine and Health Sciences, Stellenbosch University, Cape Town, South Africa

Corresponding author: N Nel (natalievdmerwe@sun.ac.za)

Background. Given the pivotal role that simulation plays in teaching students clinical skills, it is important to understand the students' perception of using simulation laboratories.

Objectives. A descriptive qualitative research design was used to determine whether participants 'believe' they have gained competence and confidence to assess a patient holistically.

Methods. Purposive sampling of 10 individual interviews and a focus group of 7 participants was drawn from primary healthcare students who successfully completed the programme the preceding year. Data were collected by 2 trained fieldworkers and transcribed by the researcher (NN). Ethical approval was obtained from the Health Research Ethics Committee, Faculty of Medicine and Health Sciences, Stellenbosch University, Cape Town, South Africa, and informed consent from the participants.

Results. The data that emerged from the data analysis were coded and categorised into themes and subthemes. The following 5 themes emerged: simulation as a teaching method; a manikin offering effective learning; confidence in clinical practice; structure of the course; and a support system. The researcher compiled a written account of the interpretations that emerged from the data analysis and verified these with the fieldworkers. Furthermore, member checking was done on 2 of the participants from the focus group and 2 of those from the individual interviews to validate the transcribed data. The findings suggest that the manikin should be upgraded regularly and be able to register a response. Data showed that the students are in favour of simulation as a foundation phase in their programme, but preferred to be introduced to a human being.

Conclusion. Simulation as a clinical teaching method ensured a good foundation phase, but students felt more competent and confident after practising on humans.

Afr J Health Professions Educ 2015;7(2):176-179. DOI:10.7196/AJHPE.363

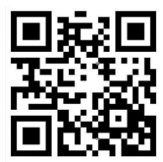

The confidence and competence levels of primary healthcare (PHC) nurse specialists are essential skills that are required when assessing a patient holistically. The PHC nurses need excellent clinical skills and require basic knowledge to think critically and analytically. Good confidence and competence levels will enable these specialists to think beyond the normal practice, be creative and innovative in finding better ways to assess and manage their patients, and ensure a safe and cost-effective practice. For PHC nurses to be confident and competent when assessing a patient, it is suggested that they are taught in a student-centred environment. ${ }^{[1]}$ Moreover, to ensure that PHC students are eventually safe and competent practitioners, it is essential that they develop their skills by practising and be declared competent before working in a clinical setting. By practising their clinical skills as a learning strategy, the students simultaneously improve their levels of competence and confidence. It was observed in the clinical field that if the confidence and competence levels of the PHC nurses are inadequate when assessing patients, they are unable to assess the patient effectively and holistically. It is a concern when assessing a patient in the clinical field, as the safe management of patients may be seriously compromised.

'Simulation is an approach to teaching and learning which is defined as a device that presents as a simulated patient (or part of a patient and interacts appropriately with the actions taken by the simulation practitioner). ${ }^{[2]}$ Educators have suggested that active learning and student participation produce better educational outcomes. ${ }^{[1]}$ According to Langlois and Thach, ${ }^{[3]}$ there is no single way of teaching in a clinical setting. Clinical teachers may adapt their styles to reflect the situations that arise. However, various educationalists describe approaches to learning in the cognitive, affective and psychomotor domains by emphasising Bloom's Taxonomy. ${ }^{[4]}$ Larkin and Burton ${ }^{[5]}$ further argue that using the framework of Bloom's Taxonomy of Educational Objectives assists staff members to critically evaluate the patient's scenario to prevent future patient complications. It measures the cognitive, affective and psychomotor domains.

Bloom's Taxonomy has long been the average framework among clinical nurse educators and staff development co-ordinators for designing learning experiences, thus providing general guidance in the development of learning objectives. ${ }^{[6]}$ Ming Su et al. ${ }^{[7]}$ state that the revised Bloom's Taxonomy provides a framework to help educators to clarify their proposed objectives and design suitable education and assessment methods. Furthermore, Harton ${ }^{[6]}$ states that learning in each domain builds on previously acquired knowledge - from simple to complex. According to Meyer and Van Niekerk, ${ }^{[4]}$ achieving the ultimate level in each domain depends on mastering previous levels. Students may not be able to solve problems if they do not know what the problem involves or how to go about solving it. Therefore, educators must systematise the actions for students so that they are able to carry them out. The planned systemisation guides educators in selecting teaching and assessment strategies. ${ }^{[4]}$

The use of a human patient simulator (HPS) provides a method by which students can participate in clinical decision-making, practise skills and observe outcomes from clinical decision-making. ${ }^{[8]}$ According to Brannan et al. ${ }^{[8]}$ it was anticipated that the use of the HPS may develop students' cognitive skills and clinical confidence levels. It is important that students are fully prepared when assessing and examining patients. Bloom's Taxonomy is the focus of cognitive, affective and psychomotor domains, which are essential approaches to learning. ${ }^{[4]}$ 
Lamb $^{[9]}$ substantiates that students' clinical confidence may increase with simulator experience, as simulation enables the student to practise skills before working with patients. To ensure that PHC students eventually become skilled and competent practitioners before entering the clinical field, it is essential to develop their skills by practising. The principal investigator (PI) observed - in the clinical field - that if the confidence and competence levels of the PHC nurses are inadequate when assessing patients, they are unable to assess patients holistically. A PHC nurse working in a clinical field must be able to apply the necessary skills and knowledge required when assessing a patient holistically. Haigh ${ }^{[10]}$ argues that simulated practice in university settings offers the potential for reflection and deep learning. A deep approach to learning is student centred and involves a search for understanding. Students must therefore think actively about what they are doing. One of the elements that fosters a deep approach is active learning, where the student is actively involved. ${ }^{[4]}$

Clinical simulation is a method of active learning that offers students a wide range of learning opportunities, including ways of applying theory to practice by bringing principles learned in the classroom to life. ${ }^{[1]}$ By using simulation as a teaching method, it prepares students to face real-life situations. According to Stefanski and Rossler, ${ }^{[1]]}$ simulation has come to the forefront as an effective teaching modality in teaching the science of nursing. It is important that students are fully prepared when assessing and examining patients. However, the PI observed that PHC students studying at our university failed to adequately acquire the required clinical confidence and competence to assess the 'real' patient. At this university these students predominantly practised and acquired their clinical skills with laboratory simulations. Many students verbalised that they did not feel competent or confident to assess patients holistically after having practised on artificial manikins. This raised serious concerns for academic lecturers who applied this teaching and learning strategy, as ultimately patient safety might seriously be compromised.

This study explored the perceptions relating to the use of laboratory simulation, a method applied to teach clinical skills to postgraduate PHC students to specifically develop their clinical confidence and competence levels as required of PHC nurses in clinical practice.

\section{Purpose of the study}

The first level of care, according to the PHC policy applicable in South Africa (SA), prescribes that it should be managed by PHC nurses without the support of medical practitioners. ${ }^{[12]}$ Access to quality public services is the rightful expectation of all SA citizens. ${ }^{[12]}$ Most of these citizens are employed in a no-work, no-pay service and therefore expect an effective and efficient service. By completing this study, the appropriate learning strategy was identified and applied to produce confident and competent PHC nurses to ultimately ensure an effective and efficient service in PHC.

The purpose of this article is therefore to describe the research based on the perceptions of students who followed the PHC postgraduate programme at a selected university in SA. As part of developing clinical competence and confidence, these students were first exposed to the use of the simulation laboratory before they had any clinical encounter with a patient.

\section{Problem statement}

With regard to the discussion above, the low confidence and inadequate competency levels demonstrated by PHC students in the clinical field, despite their interaction in a simulation laboratory, may compromise patient safety. It therefore became imperative that a scientific investigation be undertaken to explore the perceptions of postgraduate PHC students who used simulation laboratories as a learning strategy.

\section{Research question}

The following research question guided the study: 'What are the perceptions of postgraduate PHC students utilising simulation laboratories as a clinical teaching method?'

Objectives were set to determine whether participants 'believed' that they had gained competence and confidence to assess the patient holistically.

\section{Methods \\ Research design and sample}

This study followed a descriptive qualitative approach that explored the perceptions of postgraduate PHC students who were taught clinical skills utilising simulation laboratories. The target population comprised parttime PHC students who obtained the Diploma in Primary Health Care in 2010 at the university chosen for the purpose of this study in the Cape Town Metropole in SA. This university was selected purposefully as it enrolled an average of 120 students per year who followed a postgraduate diploma in PHC. This university specifically used simulation laboratories as a teaching method to teach clinical skills to these students. A purposive sample of 10 individual interviews and a focus group of 7 participants was drawn from this population of successful students. These students were purposively selected to ensure the variability of the sample. More participants would be drawn should data saturation not be achieved after the 10th interview. Data saturation was met after these interviews.

\section{Pilot study}

A pilot study was conducted on one of the participants to test the feasibility of the study; these results are not included in this study.

\section{Trustworthiness}

The trustworthiness of this study was established according to Guba and Lincoln. ${ }^{[13]}$

\section{Credibility}

Credibility was assured by being satisfied that the participants understood the questions well and agreed to the accuracy of the transcribed data. Member checking was done with 2 of the participants from the focus group and 2 from the individual interviews to have the transcribed data validated.

\section{Transferability}

A conceptual theoretical framework based on Bloom's Taxonomy and the use of more than one method of data collection strengthened transferability.

\section{Dependability}

Dependability was assured by the use of a tape recorder to ensure that all the information given by the participant was recorded. A second fieldworker took notes during the interviews with the participants. All interviews were conducted in the same manner by using an interview guide. The data were transcribed and analysed after each interview and verified by a fellow researcher, 2 fieldworkers who collected the data, and an expert in qualitative research. The researcher and fieldworkers discussed the transcribed data and clarified differences of opinion to ensure that the 
interpretation of the transcripts was congruent with the recorded interview. The analysis of the data and the themes and subthemes was further verified by the supervisor of the researcher.

\section{Conformability}

Member checking was done. Hence, the participants in this study were given an opportunity to be informed of the results of the study and make further recommendations. The participants, however, did not make any further recommendations.

\section{Data collection}

An interview guide was designed based on the objectives of the study, the literature review and the researcher's professional experiences. Two trained fieldworkers were responsible for collecting the data to prevent bias, as the researcher is a lecturer at the university. Participants were quoted verbatim in the transcriptions, as it assisted in confirming the data collected. Thereafter, the researcher read and reread the transcriptions. One of the fieldworkers had a guideline with open-ended questions, which guided the interviews in order to collect the data. The fieldworker conducted the interviews in the university's seminar room - in agreement with the participants. The participants gave signed permission that the interviews could be recorded with the use of a tape recorder. The second fieldworker, a PHC nurse, had no role in the interviewing of the participants but documented and recorded the interviews.

\section{Ethical considerations}

Consent was obtained from each participant for taking part in the study and the use of audio- and written recordings of the interviews. The participants were assured of anonymity. Permission to conduct this study was obtained from the Health Research Ethics Committee, Faculty of Medicine and Health Sciences, Stellenbosch University. All ethical principles were adhered to. Data are locked and stored in a safe place for at least 5 years - only the PI has access to the safe.

\section{Data analysis}

The transcription of the interviews was done by the researcher to familiarise herself with the data. In this manner she became familiar with the data as these were gathered. Data were analysed according to Tesch's ${ }^{[14]} 8$-step model. The data that emerged from the data analysis were coded and categorised into themes and subthemes. Coding, elaboration and recording continued until no new insights appeared. The written account of the interpretations that emerged from the data analysis was verified with the fieldworkers.

\section{Presentation of findings}

The first theme was labelled 'Simulation as a teaching method', where participants spoke willingly about their perception of simulation during their 1-year postgraduate diploma in PHC studies. A number of participants stated that the use of simulation prepared them for what to expect in the clinical setting.

'It helped me a lot, it gave me an idea how to start and where to start ... ?

'... for me I would say that simulation was the foundation of, ... but not the ultimate.

The second theme related to 'A manikin offers effective learning' The use of a manikin is one of the simulation methods the students were exposed to - they felt that the manikin was outdated. They also thought that communication was a very important aspect and wanted 'something' to communicate with them. They expressed the need to be exposed to different anatomical challenges of human beings before entering the clinical field.

'With a doll, you don't know are you working too rough or too soft, because the doll can't tell you that, they can't communicate.'

The third theme was 'Confidence in clinical practice'. Participants indicated that they gained confidence when exposed to practising on human beings. Even though they practised repeatedly on manikins, their confidence developed slowly. A few of the participants stated that their confidence developed with time. It took them a few months of practising to be confident enough to examine a patient.

'I practised on my family and that gave me confidence cause you interact with a person while you learn.'

The fourth theme was the 'Structure of programme'. When asked whether it was feasible to use simulation in PHC, all 17 participants agreed that it must be used. They verbalised the need to interact with patients at an earlier stage; hence the reason why they did not find the manikin effective as a clinical teaching method.

'If I had a choice then I would have wanted the practical to start in the clinics.'

The fifth theme consisted of the 'Support system' Simulation is a good starting point; however, students expressed the need to be introduced to a human being before entering the clinics. They took the initiative to practise on their family or friends at home to build their confidence. Most of the participants stated that the support and practice sessions among students were wonderful and benefited them in their studies.

'... we were a team, and worked as a team. We helped each other that way, if we forgot something the other one remembered it.'

\section{Discussion}

The results have shown that the use of simulation as a teaching method in postgraduate teaching of PHC nurses' skills is effective to some extent. It is pivotal that the PHC nurses are well prepared to assess a patient competently and confidently, as the safety of patients' lives are at stake. According to Ward-Smith, ${ }^{[15]}$ simulation learning is used to promote clinical competence and reflective thinking skills. The norms and standards set out by the Department of Health ${ }^{[12]}$ emphasise that PHC is at the heart of the strategy to change the health services in SA. An integrated package of essential PHC services available to the entire population will provide a solid foundation for a single, unified health system. PHC nurses, after completion of a postgraduate programme in $\mathrm{PHC}$, become independent clinical nurse practitioners in the SA context and are expected to function without the support of a doctor.

There was much debate among members of the focus group about the use of manikins as a learning method. Some of the participants felt that manikins were not needed, but agreed that they were a good starting point, while most enjoyed working with them. Most of the participants emphasised that they should be introduced to a human being, because the manikins were anatomically 'perfect' and differed from human beings, who have excess fat and skinfolds, which are absent in manikins. The participants also 
found it problematic that the manikin could not communicate verbally and non-verbally, creating a barrier in developing affective skills and obtaining subjective data during an assessment phase - a much-needed requirement when assessing patients.

To develop competence, multiple practice sessions are required to enable a student to competently assess a patient, as this is a gradual process. Furthermore, competence in assessing patients should include practising on human beings. Positive and negative opinions were obtained from the participants with regard to their perceptions of simulation laboratories. All the participants finally agreed that simulation is a 'good starting point' in the clinical programme. Moreover, they referred to simulation as a foundation phase that prepares them for what to expect when working with a patient. The participants felt that the use of simulation is positive in a clinical programme, but would have preferred to be introduced to a human being before working with patients, as the human being made them feel more confident. The use of an HPS provides a method by which students can participate in clinical decision-making and practice skills and observe outcomes from clinical decision-making. ${ }^{[8]}$ According to Brannan et al. ${ }^{\left[{ }^{[8]}\right.}$ it was anticipated that the use of the HPS may develop students' cognitive skills and confidence levels. The cognitive domain involves knowledge and development of intellectual skills, including the recognition of specific facts, procedural patterns, and concepts that serve in the development of intellectual abilities and skills. ${ }^{[16]}$ The participants felt that by practising on people individual confidence to assess a patient increased.

The results of the study are supported by Bloom's Taxonomy. Firstly, the students obtain new knowledge, which is the theory of the programme that forms part of the cognitive domain. To develop competence, theory is applied by practising on the manikin in order to become competent. During the progression of their skills the students develop the affective domain, which does not happen if only simulation is used. During practice sessions the students start contemplating new ways of becoming more skilled and competent, which supports the psychomotor domain.

The study focused on the perception of the postgraduate PHC students utilising simulation laboratories at one university. It excluded other institutions offering a similar programme. The study excluded other disciplines such as medicine and physiotherapy, which also have a clinical component in their programmes. Further research is therefore recommended in this regard.

\section{Conclusion}

The participants in the 1-year postgraduate diploma in PHC perceived the use of simulation laboratories as positive, yet felt that they should be introduced to reality at a much earlier stage of the programme. Practising on human beings as models made them more competent and confident to assess patients holistically in the clinical field. Simulation is an excellent teaching method to prepare the student in terms of what to expect and a starting point for the development of confidence, but the results showed that by practising on a human being prepared them for the clinical setting. It is suggested that the manikins should be upgraded on a regular basis and match the actual clinical setting. In this manner the use of simulation as a teaching strategy may complement the clinical setting, to which students will ultimately be exposed.

Acknowledgements. The authors would like to thank the students who agreed to participate in this study.

\section{References}

1. Haider E. Clinical simulation: A better way of learning? Nurs Manag (Harrow) 2009;16(5):22-23.

2. Gaba DM.The future vision of simulation in health care. Quality and Safety in Health Care 2007;12(1):2-10. 3. Langlois J, Thach S. Teaching and learning styles in the clinical setting. Family Medicine 2001;33(5):344-346. 4. Meyer S, van Niekerk S. Nurse Educator in Practice. Cape Town: Juta, 2008.

5. Larkin BG, Burton FJ. Evaluating a case study using Bloom's Taxonomy of Education. AORN J 2008;88(3):390402. [http://dx.doi.org/10.1016/j.aorn.2008.04.020]

6. Harton BB. Clinical staff development: Planning and teaching for desired outcomes. J Nurses Staff Dev 2007:23(6):260-268.

P. Starnes B. Applying the revised Bloo is Taxonomy to a medicat surgial norsing lesson.

Nurse Educ 2004;29(3):116-120. 8. Brannan JD, White A, Bezanson JL. Simulator effects on cognitive skills and confidence levels. J Nurs Educ 2008; $47(11): 495-500$

9. Lamb D. Could simulated emergency procedures practices in a static environment improve the clinical performance of a Critical Air Support Team (CCAST)? A literature review. Intensive and Critical Care Nursing 2007;23:33-42.

10. Haigh J. Using simulation to prepare students for interprofessional work in the community. J Nurs Edu 2007;7(2):95-102

11. Stefanski RR, Rossler KL. Preparing the novice critical care nurse: A community-wide collaboration using the benefits of simulation. J Contin Educ Nurs 2009;40(10):443-451. [http://dx.doi.org/10.3928/00220124-20090923-03]

12. Department of Health. The Primary Health Care Package for South Africa - a Set of Norms and Standards. Pretoria: Government Printer, 2000. http://www.doh.gov.za/docs/policy/norms/full-norms.html (accessed 2 May 2011).

13. Guba EG, Lincoln YS. Fourth Generation Evaluation. Beverly Hills: Sage, 1985.

14. Tesch R. Qualitative Research: Analysis Types and Software Tools. Bristol: Farmer, 1985.

15. Ward-Smith P. The effect of simulation learning as a quality initiative. Urol Nurs 2008;28(6):471-473.

16. Clark DR. Big Dog \& Little Dog's Performance Juxtaposition: Bloom's Taxonomy of Learning Domains, 2010. http://www.nwlink.com/ donclark/hrd/bloom.html (accessed 2 May 2011). 